\title{
DEPENDENCE OF SHAPE ON PARTICLE SIZE FOR A CRUSHED ROCK RAILWAY BALLAST
}

L.M. Le Pen, W. Powrie, A. Zervos, S. Ahmed, S. Aingaran

University of Southampton, Southampton, Hampshire, United Kingdom

ABSTRACT

Laboratory testing of railway ballast poses practical difficulties because the particle size is often too large for most standard apparatus. There are therefore advantages in developing a scaled material whose behavior is representative of the full size material. A first stage in validating such an approach is to investigate whether the particle shape is affected by the change in scale. This paper sets out methods for evaluating form and roundness (aspects of shape) and proposes a new measure for evaluating roundness, termed ellipseness. These methods are then applied to a crushed rock railway ballast over a range of particle sizes. Statistical analysis demonstrates a measurable variation in the distributions of form and roundness with particle size over a range of sieve intervals, although the differences are slight and do not necessarily rule out the use of a scaled material for investigating the factors influencing macro mechanical behavior.

Keywords: Ballast, shape, form, statistics, weibull, scale, roundness, angularity, ellipseness

\section{Introduction}

The application of soil mechanics principles to the mechanical behavior of railway ballast should lead to improved ballast specifications and track substructure designs, and reduced maintenance requirements and whole life cost. However, a significant difficulty in carrying out experimental investigations into the behavior of railway ballast is the typical particle size (up to $62.5 \mathrm{~mm}$ ), which is too large for a representative specimen to be accommodated in most standard laboratory soil element testing apparatus. Large scale apparatus has been developed (e.g.[1], [2], [3]), but is expensive to build and run and tests can be challenging and time-consuming. 
An alternative approach is to develop and test a scaled material having the same characteristics as railway ballast but a small enough particle size to be tested in standard laboratory apparatus. A potential concern is the preservation of particle properties with scaling; for example, particle shape has been shown to influence the bulk density, stiffness and strength of natural and crushed sands (e.g. [4]). Historically, research into scaled particulate materials was in the context of rockfill (e.g. [5]) but recent research has been driven by the need to understand the behavior of railway ballast (e.g. [6]). However, despite some notable efforts there have been few systematic attempts to investigate the use of appropriate scaled materials. Such an investigation would require consideration of the shape (form, roundness and roughness), stiffness (contact and material) and strength (fracture toughness and abradability) of particles; how these attributes vary within and over a range of particle sizes; and how any variation affects the macromechanical behavior.

This paper describes an investigation to measure and evaluate how particle shape may change with scale. The same parent rock is used, minimizing the potential for variations in surface roughness does not vary; the focus is therefore on comparing the form and roundness over a range of particle sizes in convenient sieve intervals from full size to reduced scale.

In particular the paper

1. develops appropriate methods for defining and quantifying particle shape,

2. shows that there are measurable and quantifiable, although small, differences in particle shape with size.

The detailed measurements of shape will also facilitate numerical simulation using particles that represent crushed rocks by taking advantage of recent advances that permit particles of arbitrary shape to be used (e.g. [7]).

\section{Background}

Two principal approaches have been proposed to create of an analogous or model material at a smaller scale. They are:

- scalping (e.g. [8]), i.e. the removal of all particles from the original material that are greater than a certain size; 
- parallel gradation (e.g. [9]), i.e. the formation of a laboratory specimen with a particle size distribution smaller than but parallel to that of the original material.

The feasibility of using either method may depend on the material being tested. For example scalping might be appropriate for a well graded material where relatively few oversize particles need to be removed. However, for a more uniformly sized material such as a railway ballast, scalping might result in the removal of much of the specimen so the parallel gradation technique would be more appropriate.

The validity of the parallel gradation approach relies on the smaller scale particles behaving in the same way as the larger scale particles they are intended to model. [5], [6], and [10] investigated this by means of triaxial tests on rockfills over a range of parallel gradations, in triaxial cells of varying size. In general all three studies demonstrated that the volumetric strain varied with scale for tests under the same stress conditions. However it is not clear if these trends resulted from changes in shape or changes in other characteristics related to scale. [6] also compared particle shapes for the three parallel gradations of Iron Mountain Trap Rock from Missouri in the USA tested on the basis of plan view images. Although only averages of the data on particle shape were reported, they showed that larger particles tended to have greater aspect ratios (maximum to minimum dimensions, or form) and sharper corners (greater angularity, defined as the average radius of the smallest four corners within a particle).

Scale is known to influence the strength of individual particles, with smaller particles being statistically likely to be stronger owing to their lower probability of containing flaws (see e.g. [11], [12]). Hence assemblages of smaller particles may appear to be less compressible than assemblages of larger particles, if the stress applied is sufficient to fracture some of the larger particles.

The applied boundary stresses should be the same in corresponding tests on full size and scaled material. If it is assumed that the particle shape remains similar and that Hertzian (elastic) contact theory (see [13], [14]) can be applied, then the interparticle contact stresses and hence compressibility of the assembly will remain the same. In practice there may be some differences: Hertzian contact theory assumes that each surface is topographically smooth on both the micro and macro scale, and in reality there is also likely to be some inelastic behavior [15]. 
Experimental work to establish the importance of changes in shape on the behavior of soils (without varying scale) is still at a relatively early stage. Research findings are generally restricted to changes in behavior resulting from large changes in shape characteristics. For example [16] established that the relative proportions of bulky or platy particles (extremes of shape) had a strong influence on the shear strength and deformation characteristics of sands. [4] explored the impact of particle shape on sandy soils by comparing data from published studies; they too considered relatively large changes in shape to identify a number of trends in soil behavior.

\section{Brief review of Particle Shape characterization approaches and chosen measures}

In this section the historical development of ideas related to shape and some of the more established methods are briefly discussed. Full details may be found in the references provided.

\section{Overview}

It is widely recognized that there are three independent properties of particle shape- form, roundness and surface texture - which have quite different scales as shown in 2D in Figure 1 [17]. These properties are independent because each can vary widely without influencing the others. Accepting these broad definitions, it is then necessary to come up with means of measuring or characterizing each of the three properties. For form and roundness this has usually meant combining measurements into a dimensionless index, but taking measurements is not trivial and it is usually necessary to take measurements from $2 \mathrm{D}$ images or $3 \mathrm{D}$ data cloud representations of particles rather than the physical objects themselves.

Particle imaging has traditionally been based on 2D projections (e.g. using camera lucida [18]), but contemporary imaging now uses digital cameras for 2D (e.g. [19]) and laser scanning or computer tomography (CT) scanning for 3D (e.g. [20, $21,22,23])$. However, there are issues with the interpretation of such data both in $2 \mathrm{D}$ and $3 \mathrm{D}$, mainly related to the relative particle to pixel or voxel size. Also, while it might be supposed that $3 \mathrm{D}$ measures would be superior to $2 \mathrm{D}$ ones this largely depends on the reasons for making the measurements. Furthermore laser and CT scanning is not readily available to most researchers or practitioners and 
CT scanning in particular is costly in terms of both money and time. Also, with CT analysis there are physical limits on the sizes of samples that can be scanned and the resolution achievable. Therefore the methods adopted for this paper are essentially pseudo 3D measures of 2D digital images with the advantage that these methods are readily available to all researchers and practitioners working in this area and are appropriate for the aims of this work.

Some of the better known measures for form and roundness and the measures chosen for use in this research are discussed below. Also, where appropriate, issues with the use of digital images are highlighted. Roughness is not considered as this is reasoned to be similar for all of the particles evaluated owing to their method of manufacture (crushing). Therefore the pixel size used for imaging has been set to a size larger than that needed to capture this smallest scale aspect of shape.

\section{Form: measures}

Form is the largest-scale particle property. Most quantifications of form are based on measuring the longest $(\boldsymbol{L})$, shortest $(\boldsymbol{S})$ and intermediate $(\boldsymbol{I})$ orthogonal dimensions and combining two or all of them into a dimensionless index. $\boldsymbol{L}, \boldsymbol{S}$ and I can be obtained or approximated from 2D images as shown in Figure 2. One of the earliest attempts at quantifying form was by Zingg [24], who as long ago as 1935 used the position of a particle on a plot of $\boldsymbol{I} / \boldsymbol{L}$ against $\boldsymbol{S} / \boldsymbol{I}$ to classify it as either flat, columnar, spherical or flat-columnar (Figure 3). $\boldsymbol{I} / \boldsymbol{L}$ and $\boldsymbol{S} / \boldsymbol{I}$ are sometimes termed the flatness and elongation ratios respectively. Another commonly used index is the $\boldsymbol{S} / \boldsymbol{L}$ ratio, termed the degree of equancy [25]. The $\boldsymbol{S} / \boldsymbol{L}$ ratio is the product of the coordinates in the Zingg plot $(\boldsymbol{I} / \boldsymbol{L}$ and $\boldsymbol{S} / \boldsymbol{I})$. Lines of equal $\boldsymbol{S} / \boldsymbol{L}$ are hyperbolae on the Zingg plot and each may pass through up to three of the classification sectors. Measures that include all three of the parameters $\boldsymbol{S}, \boldsymbol{I}$ and $\boldsymbol{L}$ have appeared in the literature (e.g. [26]). However there are ambiguities because two of the parameters (and hence the form) can vary and still yield the same result. In this sense they are generally less effective than the simpler $\boldsymbol{S} / \boldsymbol{L}$ ratio which at least retains some link to a shape type. Identifying orthogonal axes coincident with $\boldsymbol{S}, \boldsymbol{I}$ and $\boldsymbol{L}$ and measuring these dimensions for large numbers of particles presents practical difficulties. It is possible to measure $\boldsymbol{S}, \boldsymbol{I}$ and $\boldsymbol{L}$ manually using calipers, although this is time 
consuming and any set of measurements may be subject to user variation. There are now various algorithms that may be implemented using 2D digital images or 3D data clouds. However, there are issues with automated methods and in choosing a method consideration should be given to the types of shape being evaluated and how the algorithm might distort the intended measure (e.g. [27]). Pseudo 3D measurements can be generated using orthogonal views and can be just as good as estimates gained from 3D data. For example [23] compared using 3D representations of particles (from CT or laser scanning) to using orthogonal 2D images to determine form and concluded that for 2D and 3D methods ". No one is consistently better than the other ....". A technique that will be adopted for this research was put forward by [28]. This technique relies on allowing the particles to fall onto a flat horizontal surface, on the basis that the particles will tend to come to rest with their shortest dimension $(\boldsymbol{S})$ upward and their longest and intermediate dimensions visible in plan $(\boldsymbol{I}$ and $\boldsymbol{L})$. Thus digital plan view images could be used to measure $\boldsymbol{I}$ and $\boldsymbol{L}$. By assuming that the shape of the particle would correspond approximately to a scalene ellipsoid (i.e., an ellipsoid where the three major axes have different lengths) and measuring the mass $(\boldsymbol{W})$ of the particle, $\boldsymbol{S}$ can be estimated for single or groups of particles using Equation 1:

$$
S=\left(W / \rho_{w} G_{s}\right)(6 / \pi L I)
$$

where $\boldsymbol{G}_{\boldsymbol{s}}$ is the specific gravity of the particle (for granite typically 2.65) and $\boldsymbol{\rho}_{\boldsymbol{w}}$ is the density of water $\left(1000 \mathrm{~kg} / \mathrm{m}^{3}\right.$ at $\left.4^{\circ} \mathrm{C}\right)$.

This approach to estimating $\boldsymbol{S}$ from measurements of $\boldsymbol{L}, \boldsymbol{I}$ and $\boldsymbol{W}$ is termed the scalene ellipsoid equivalent sphericity, or SEES. [28] validated the approach by taking orthogonal views of a number of particles of varying shapes and comparing the ratio of $\boldsymbol{S} / \boldsymbol{L}$ obtained from each method. Of the materials they tested, Leighton Buzzard sand (fraction B) is geometrically the most similar to railway ballast (albeit at smaller scale) for which the averaged measured $\boldsymbol{S} / \boldsymbol{L}$ of 0.532 compared well with the calculated value (using equation 1) of 0.549 for the six particles individually measured and weighed. A more detailed description and a validation of the SEES approach by comparison to orthogonal views for the ballast material, are given in the section on materials and methods. 


\section{Roundness: measures}

Roundness is the intermediate scale descriptor of shape, commonly considered the inverse of angularity in that as one increases the other decreases. Unlike form there is no direct physical method of measurement for roundness. Roundness has most usually been quantified using $2 \mathrm{D}$ views of particles, but 3D methods have appeared in the literature [29]. Measures can be complex and subjective in application. Probably the most well-known method was put forward by Wadell [18], in which the radius of identifiable corners in the particle outline are measured and averaged and then divided by the radius of the maximum inscribed circle. In effect this is a measure of average angularity. Another well-known method was put forward by Krumbien [30], this uses comparison charts to classify particles by eye. A version of this type of chart can be found in [31] where shapes are shown in a 4 by 5 array with progressively increasing roundness [18] across the horizontal scale and aspect ratio (form) on the vertical scale. It is extremely time consuming to carry out either of these procedures manually, and while some progress has been made in automating Wadell's methods (e.g. [32]) there remain difficulties in defining what constitutes a corner. Furthermore the use of predrawn reference charts is more suited to the classification of different materials rather than examining particles from the same source material (as is the case in this research), where differences in shape with scale may be too small to register. In this research we have chosen to use a relatively simple method easily adapted to automatic digital image analysis, based on quantifying the ratio of the perimeter $(\boldsymbol{P})$ of the 2D projection of a particle to the perimeter of a reference shape of the same area $(\boldsymbol{A})$ i.e. $\boldsymbol{A}_{\boldsymbol{o}}=\boldsymbol{A}_{\boldsymbol{e}}$ but $\boldsymbol{P}_{\boldsymbol{o}} \neq \boldsymbol{P}_{\boldsymbol{e}}$. The subscripts $\boldsymbol{o}$ and $\boldsymbol{e}$ denote respectively the particle (object) and the reference shape, assumed here to be an ellipse.

In the current research, for consistency with the reference shape adopted in the analysis of form, we use a new measure of roundness that we call "ellipseness" $\boldsymbol{E}$, defined as:

$$
E=\frac{\text { Perimeter of equivalent area ellipse }}{\text { Perimeter of particle }}=\frac{P_{e}}{P_{o}}
$$

In this calculation $\boldsymbol{E}$ will always be less than 1, meaning that the perimeter of the actual particle is greater than that of an ellipse of the same area. As the calculated value of $\boldsymbol{E}$ approaches 1, the particle becomes more ellipse like. This has some similarity to the commonly used "equivalent disc method", in which the reference 
shape is a circle of the same area. However, using a circle would imply an $\boldsymbol{I}$ dimension greater than is physically possible. The use of an ellipse having the same major dimension and area provides a better estimate of the smooth shape used to describe the particle. That particles do not all tend to circles when perfectly round was noted by Wadell as long ago as 1932 [18]. Methods of determining $\boldsymbol{E}$ using digital images are discussed in the section on materials and methods. It is recognized that ellipseness is an average measure of roundness (or inverse angularity) as the same numerical value may be achieved in a variety of ways (e.g. two very sharp projections/corners on a notionally elliptical 2D image may result in the same ellipseness as a number of smaller, less pronounced, projections/corners).

\section{Materials and methods}

\section{Materials}

Full size and reduced scale crushed granite ballast was obtained from Cliffe Hill Quarry, Leicestershire, England which supplies ballast for Network Rail. Most ballast around the world is formed of particles within the range $22.4 \mathrm{~mm}$ to 63.5 $\mathrm{mm}$. For this study the lower limit of the particle range is extended to $9.5 \mathrm{~mm}$ so that the scaled and full size particles can be grouped over a sequential range of eight sieve intervals as follows: (1) $9.5 \mathrm{~mm}$ to $11.2 \mathrm{~mm}$, (2) $11.2 \mathrm{~mm}$ to $13.2 \mathrm{~mm}$, (3) $13.2 \mathrm{~mm}$ to, $16.0 \mathrm{~mm}$ and (4) $16.0 \mathrm{~mm}$ to $22.4 \mathrm{~mm}$ (scaled), (5) $22.4 \mathrm{~mm}$ to $31.5 \mathrm{~mm}$, (6) $31.5 \mathrm{~mm}$ to $40.0 \mathrm{~mm}$, (7) $40.0 \mathrm{~mm}$ to $50.0 \mathrm{~mm}$, and (8) $50.0 \mathrm{~mm}$ to $62.5 \mathrm{~mm}$ (full size). The smaller sieve size in each interval will be referred to as the catching sieve and the larger the passing sieve. For ease of reference, often only the catching sieve size will be stated.

Figure 4 shows example particles from each sieve interval; the images have been resized so that the particle sizes appear to be approximately the same. The Figure is provided as a visual demonstration that differences in shape with scale appear to be slight and could be subjectively described as not visually appreciably different. For example if the comparison chart provided by [31] were applied the particles could easily be placed in the same classification sector. 


\section{Imaging system: methods}

Groups of particles were placed on a bench top with a digital camera directly above as shown in Figure 5. The camera was positioned approximately $1.2 \mathrm{~m}$ above the particles and the particles placed within an approximately $200 \mathrm{~mm}$ sided square immediately below the camera. The focal length of the camera was set to $120 \mathrm{~mm}$. The distortion of objects not directly below the camera was measured and found to be insignificant at up to $100 \mathrm{~mm}$ lateral offset. The camera used is a SONY DSC-R1 Cyber shot 10 mega pixels with a $21.5 \mathrm{x}$ $14.4 \mathrm{~mm}$ (max image size 3888 x 2592) CMOS sensor having a pixel density of 4593dpi horizontally and 4572dpi vertically. This research looks at particles of relatively large size (diameters mainly between $10 \mathrm{~mm}$ to $60 \mathrm{~mm}$ ) and the properties of the camera used were suitable for this work as would those of many commonly available modern cameras.

The particles were lit by a pair of 200 watt stroboscopic daylight balanced lamps. Parabolic light modifiers were used to focus the light on to the sampling area. The positions of the lights are set so as to minimise/eliminate shadows in the plane being photographed. Additionally, a black background was used.

Off the shelf image analysis software was used to segment and measure the images using consistent histogram based thresholding. The software used was Image Pro Plus [33], but there are many other suitable software packages available and/or bespoke code could be written. Digital measurements are discussed later.

\section{Form: methods}

Two different methods of measuring $\boldsymbol{S}, \boldsymbol{I}$ and $\boldsymbol{L}$ to determine form parameters were compared. In the first method, two images of the particle were taken in two different orthogonal projection planes capturing the longest, intermediate and shortest dimensions. The first view captured was of the plane in which the particle was mechanically most stable thus presenting the longest $(\boldsymbol{L})$ and intermediate $(\boldsymbol{I})$ particle dimensions to the camera. Each particle was then rotated and the orthogonal view eliciting the shortest dimension identified. From the images thus obtained, image analysis was used to determine the lengths of the longest $\left(\boldsymbol{D}_{\max }\right)$ and shortest $\left(\boldsymbol{D}_{\min }\right)$ lines joining two points on the particle perimeter and passing through its centroid of area (Figure 6). If, as is the case here, the shapes analysed 
are reasonably regular, these measurements approximate to $\boldsymbol{L}, \boldsymbol{I}$ and $\boldsymbol{S}$. $\boldsymbol{L}$ and $\boldsymbol{S}$ were taken as the absolute maximum and minimum values of $\boldsymbol{D}_{\boldsymbol{m a x}}$ and $\boldsymbol{D}_{\boldsymbol{m i n}}$ respectively, and $\boldsymbol{I}$ as $\boldsymbol{D}_{\min }$ from the first most stable projection.

The second method involved determining $\boldsymbol{L}$ and $\boldsymbol{I}$ from the initial plan image of each particle, and inferring the value of $\boldsymbol{S}$ using the SEES approach (Equation 1). Systematic comparison of the results obtained using the two methods showed that in a minority of cases (less than $9 \%$ for the scaled material and less than $4 \%$ for the full size material), the plan view image gave $\boldsymbol{L}$ and $\boldsymbol{S}$ rather than $\boldsymbol{L}$ and $\boldsymbol{I}$ so that applying SEES gave $\boldsymbol{I}$ rather than $\boldsymbol{S}$. In these cases the values $\boldsymbol{S}, \boldsymbol{I}$ and $\boldsymbol{L}$ were reassigned accordingly. This problem occurred because while the ballast particles approximate reasonably well to scalene ellipsoids, some particles have $\boldsymbol{S} \approx \boldsymbol{I}$ and the most stable face is not clearly identifiable.

\section{Roundness: methods}

The area and perimeter were measured from the digital images (as described and discussed in the next section). With these values known, Equation 2 was used to quantify roundness by means of the Ellipseness measure as follows. The minor radius $\boldsymbol{b}$ of the equivalent area ellipse was calculated from the major radius $\boldsymbol{a}=$ $\boldsymbol{L} / 2\left(\boldsymbol{D}_{\boldsymbol{m a x}} / 2\right)$ and the measured area $\boldsymbol{A}_{\boldsymbol{o}}$ using Equation 3. The equivalent perimeter $\boldsymbol{P}_{\boldsymbol{e}}$ was then calculated using Equation 4.

$$
\begin{aligned}
& b=\frac{A_{o}}{\pi a} \\
& P_{e} \approx \pi(a+b)\left(1+\frac{3\left(\frac{a-b}{a+b}\right)^{2}}{10+\sqrt{4-3\left(\frac{a-b}{a+b}\right)^{2}}}\right)
\end{aligned}
$$

Equation 4 was first presented in 1913 by the mathematician Ramanujan [34] and is an accurate approximation to the perimeter of an ellipse (which can otherwise be calculated using power series). A discussion on approximations to the perimeter of an ellipse can be found in [35]. 


\section{Influence of digitization on the measured parameters}

In image analysis it is important to define precisely the methods used and evaluate the suitability of the pixel density (i.e. the number of pixels per particle) used. In this study the camera set up was such that each pixel represented approximately $1 / 8 \mathrm{~mm}$. This was selected so as to remove the effects of surface roughness from the measured perimeters while still providing sufficient pixel density, in all particles analysed, not to unduly influence the measured diameters, perimeters and areas used for the calculation of form and ellipseness. The pixel density over the size range of ballast particles imaged was approximately 10,000 to 300,000 pixels per particle.

The diameters $\boldsymbol{D}_{\max }$ and $\boldsymbol{D}_{\min }$ are defined in Figure 6 and the associated text. Area measurement is by summation of pixels and it has been shown that this method usually converges after only several hundreds of pixels (e.g. [27]). Therefore with 10,000 pixels or more the measured areas can be considered reliable. The method used to measure the perimeter is "eight point connect" (sometimes termed "polygonal"), which for rough objects is known to give digital perimeters that increase with pixel density.

To investigate the influence of the pixel density on the measurements made in this study - and in particular to evaluate whether the measurements of perimeter would have sufficient pixels present to capture particle roundness - smooth irregular objects, (i.e. objects having no measurable surface roughness for the pixel densities applied) including some of similar form and ellipseness to ballast were imaged over the range of pixel densities used (achieved by repositioning the camera). This investigation resulted in the following observations:

- Form is a dimensionless ratio of combinations of $\boldsymbol{L}, \boldsymbol{I}$ and $\boldsymbol{S}$ ( $\boldsymbol{D}_{\max }$ and $\boldsymbol{D}_{\boldsymbol{m i n}}$ ). It can therefore be calculated using the number of pixels present and the true scale is irrelevant. This has the advantage that calibration of images to true scale is not needed, and this potential small source of error can be discounted. The investigation confirmed that form was substantially insensitive to the range of pixel density used, (i.e. the aspect ratio thus calculated typically varied by less than $1.0 \%$ ).

- Ellipseness was calculated using the measurements of $\boldsymbol{L}\left(\boldsymbol{D}_{\max }\right)$ area and perimeter. Again ellipseness is a dimensionless ratio that may be calculated using measurements in pixel units rather than true scale. 
Calculations demonstrated that ellipseness was also substantially insensitive for the range of pixel densities used (i.e. for ellipseness, as the pixel density reduced over the full range ellipseness typically increased by not more than $0.5 \%$ ).

\section{Measurements of particle shape, results, analysis and discussion}

Form

Values of the ratios $S / L, I / L$ and $S / I$ measured on the basis of two orthogonal views are compared with those estimated from the plan view using the SEES approximation, for particles retained on each sieve size, in Table 1.

Figure 7a shows a Zingg plot for particle form data determined using SEES, while Figure $7 \mathrm{~b}$ shows data for the same particles determined using two orthogonal views. A comparison of the $\boldsymbol{S} / \boldsymbol{L}$ ratio obtained from each of the methods is shown in Figure 8. Estimates of $\boldsymbol{S}$ for an individual particle and hence its position on the Zingg plot can vary greatly. However, taken overall these variations average to a much smaller error, approximately a $2 \%$ underestimate in $\boldsymbol{S} / \boldsymbol{L}$ by SEES compared with using two orthogonal views for most particle sizes, with bigger errors of $7.7 \%$ and $-10.2 \%$ respectively for the $31.5-40.0$ and $>40.0 \mathrm{~mm}$ particle size ranges. Further measurements were then made using the SEES approach, increasing the total number of particles measured to 875 (Table 2). Figure 9(a) shows a Zingg plot for all data sorted by sieve interval and Figure 9(b) shows the average position on the graph for each sieve interval. Figure 9(b) shows that on average the data are all very near to the crossover between the four particle classification sectors $(2 / 3,2 / 3)$. The particles on the $11.2 \mathrm{~mm}, 13.2 \mathrm{~mm}$ and 16.0 $\mathrm{mm}$ catching sieves classify as "spherical" and those on the $50.0 \mathrm{~mm}$ catching sieve just cross into the "flat" region. The particles on the $9.5 \mathrm{~mm}, 22.4 \mathrm{~mm}$ and $31.5 \mathrm{~mm}$ catching sieves are in the "columnar" sector and those on the $40.0 \mathrm{~mm}$ catching sieve are in the "flat and columnar" region. The nearness to the crossover may tend to over emphasize differences that in reality are slight. Table 3 summarizes key statistics from the $\boldsymbol{I} / \boldsymbol{L}, \boldsymbol{S} / \boldsymbol{I}$ data shown in the Zingg plots and also the $S / L$ data for all particles.

Having considered the effect of all three parameters $\boldsymbol{S}, \boldsymbol{I}$ and $\boldsymbol{L}$ in the Zingg plots, the $\boldsymbol{S} / \boldsymbol{L}$ ratio will now be investigated further. Figure 10 plots separately the cumulative distributions of $\boldsymbol{S} / \boldsymbol{L}$ ratio for the particles in each sieve interval. The 
curves for full size ballast group together on the left. All other distributions form a second band to the right, with the exception of the data for the $9.5 \mathrm{~mm}$ catching sieve curve which falls between the two bands.

The distributions shown in Figure 10 may be compared statistically by means of a Kruskal Wallis analysis [36]. This uses the rank of a variable to determine the statistical likelihood that separate data sets have the same median. In Figure 11, the rank (i.e. the relative position of each individual $\boldsymbol{S} / \boldsymbol{L}$ value within the full data set of 875 particles) is plotted on the $\mathrm{x}$ axis. The groups designated by catching sieve size are indicated on the y axis. For each group, a line showing the $95 \%$ confidence interval for the median has been drawn. Where there is no overlap between the confidence intervals, there is at least a $95 \%$ confidence that the medians of the two groups are different. In Figure 11, the 95\% confidence limits for the $9.5 \mathrm{~mm}$ catching sieve group are indicated by vertical lines which helps to illustrate that there are two possible groupings with overlapping $95 \%$ confidence intervals denoted by catching sieve groups as:

- $9.5 \mathrm{~mm}, 22.4 \mathrm{~mm}, 31.5 \mathrm{~mm}, 40 \mathrm{~mm}$ and $50 \mathrm{~mm}$

- $11.2 \mathrm{~mm}, 13.2 \mathrm{~mm}$ and $16 \mathrm{~mm}$

The Kruskal Wallis analysis supports the groupings observed in the cumulative particle size distributions shown in Figure 10.

As well as comparing the measured data (Figures 9 and 10) it is possible to fit equations to these distributions. Providing mathematical functions that capture the range and relative distribution of shapes within each size allows others to make comparisons with this data. Statistical confidence limits to the distributions can also be determined. However, there is no fundamental reason why any particular function should be used to fit the shape measurements and it is therefore reasonable to use functions of wide versatility (i.e. able to fit a range of distribution types). A Weibull distribution fits this description and has a record of application to particle properties_(e.g. particle crushing strength [11]). The Weibull cumulative distribution function (CDF) is given by:

$$
C D F=y=1-e^{-\left(\frac{x}{a}\right)^{b}}
$$

The Weibull probability density function (PDF) is given by:

$$
P D F=y=b a^{-b} x^{b-1} e^{-\left(\frac{x}{a}\right)^{b}}
$$


Where $\boldsymbol{a}$ is the scale parameter and $\boldsymbol{b}$ is the shape parameter, both found by obtaining a best fit to the sample data [37]. The Weibull distribution is very similar to the symmetrical normal distribution (PDF) when the shape parameter $\boldsymbol{b}$ is between 3 and 4; as $\boldsymbol{b}$ increases above 4 the curve skews to the right. When $\boldsymbol{b}=1$ the Weibull distribution reduces to the exponential distribution and when $\boldsymbol{b}=2$ to the Rayleigh distribution. The scale parameter $\boldsymbol{a}$ serves to smear the function along a greater or lesser length on the x-axis, reducing or raising the relative peak. Equation 5 can be used to match the cumulative distribution curves shown in Figure 10. A least squares fit to the $\boldsymbol{S} / \boldsymbol{L}$ data for each sieve interval, performed using the software Matlab [38], gave the values shown in Table 4 for the parameters $\boldsymbol{a}$ and $\boldsymbol{b}$. Figure 12 shows the Weibull fits for the cumulative distribution functions for the $\boldsymbol{S} / \boldsymbol{L}$ data from each catching sieve size. Comparison of Figure 12 with Figure 10 shows that the Weibull functions are generally close to the measured data. Figure 13 shows the Weibull fit for the probability density function for the $\boldsymbol{S} / \boldsymbol{L}$ data for each catching sieve size which again shows the differences between the catching sieve groupings. The data shows that there is a weak trend for $\boldsymbol{S} / \boldsymbol{L}$ to reduce for larger particles (as found by [6]), but there are exceptions. Some of the data are more spread out, indicated by a greater standard deviation (Table 3) and or flatter looking probability density function (Figure 13) such as the data in the catching sieve intervals $11.2 \mathrm{~mm}, 13.2 \mathrm{~mm}$ and $16 \mathrm{~mm}$. These observations are not necessarily common to the other form indicators $(\boldsymbol{I} / \boldsymbol{L}$ and $\boldsymbol{S} / \boldsymbol{I}$ ) which can be seen by comparing the values in Table 3 .

\section{Roundness}

For the dataset shown in Table 2, Image Pro Plus was used to estimate $\boldsymbol{L}\left(\boldsymbol{D}_{\max }\right)$, the perimeter $\boldsymbol{P}_{\boldsymbol{o}}$ and the area $\boldsymbol{A}_{\boldsymbol{o}}$ of each particle, from which the ellipseness was calculated using Equations 7, 8 and 9. Basic statistics for the ellipseness data is shown in Table 5. Figure 14 shows the cumulative distributions of ellipseness for each sieve interval; as with form, the curves cover a range of ellipseness values. Figure 15 presents a Kruskal Wallis analysis of ellipseness. Vertical lines are shown at the $95 \%$ confidence limits for the $22.4 \mathrm{~mm}$ data set. Four possible groupings with overlapping 95\% confidence intervals are apparent denoted by catching sieve groups as:

- $9.5 \mathrm{~mm}, 11.2 \mathrm{~mm}, 13.2 \mathrm{~mm}$ 
- $16 \mathrm{~mm}, 22.4 \mathrm{~mm}, 31.5 \mathrm{~mm}, 50 \mathrm{~mm}$

- $22.4 \mathrm{~mm}, 31.5 \mathrm{~mm}, 40 \mathrm{~mm}, 50 \mathrm{~mm}$

- $\quad 11.2 \mathrm{~mm}, 22.4 \mathrm{~mm}$ (just)

These groupings are different from those identified for form.

Again applying a Weibull fit gives the values of parameters $\boldsymbol{a}$ and $\boldsymbol{b}$ shown in Table 6 .

Figure 16 shows the CDF calculated using the Weibull functions for ellipesness.

Figure 17 shows the Weibull PDFs which are severely skewed by the high shape factor $(\boldsymbol{b})$.

The ellipseness analysis again demonstrates measurable differences in roundness between particles within each sieve range. The data in general suggests that there is a tendency for larger size particles to be more angular - again, consistent with [6]. However, it is still possible for comparisons between particular sieve intervals to counter this trend. The actual and idealized distributions of particle aspect ratio $\boldsymbol{S} / \boldsymbol{L}$ and ellipseness (angularity) could be used as checks to aid in the creation of numerical specimens for particle level discrete element analysis, for example using the approach proposed by [39] and developed by [7] or other codes capable of generating arbitrary shapes such as [40].

\section{Conclusions}

It has been demonstrated that the SEES approach to determining $\boldsymbol{S}, \boldsymbol{I}$ and $\boldsymbol{L}$ is reasonably consistent with a two-view approach for the ballast investigated and has the advantage of being less time consuming.

A new measure of roundness (termed ellipseness) has been introduced. This relates the perimeter of the particle to the perimeter of an idealized elliptical particle of the same area, and can be determined automatically using appropriate imaging and analysis software.

The data indicate a weak trend in the ballast investigated for larger particles to have a lower $\boldsymbol{S} / \boldsymbol{L}$ and greater angularity, although comparison between any two individual sieve intervals may not follow these trends. However, the ranges of variation in $\boldsymbol{S} / \boldsymbol{L}$ and angularity are relatively small in magnitude, and do not necessarily rule out the use of scaled materials as appropriate substitutes for testing purposes. 
Further work is needed to evaluate whether the small changes in shape measured in this research translate into differences in macromechanical behavior that are attributable to shape alone. Such further work might involve the use of particle scale discrete element analysis, in which case the distribution functions presented in this paper could be used as checks to aid in the creation of numerical specimens.

\section{ACKNOWLEDGEMENTS}

This research was facilitated by a grant from the Engineering and Physical Sciences Research Council for the project titled "Development and role of structure in railway ballast" (Reference: EP/F062591/1). We also acknowledge the work of Ben Powrie in carrying out particle imaging and Andrew Cresswell for his contributions to the original research proposal.

\section{LIST OF SYMBOLS}

\begin{tabular}{|l|l|}
\hline $\boldsymbol{L}$ & Longest dimension \\
\hline $\boldsymbol{I}$ & Intermediate dimension \\
\hline $\boldsymbol{S}$ & Shortest dimension \\
\hline $\boldsymbol{G}_{\boldsymbol{s}}$ & Specific gravity \\
\hline $\boldsymbol{\rho}_{\boldsymbol{w}}$ & Density of water \\
\hline $\boldsymbol{W}$ & Mass of particle \\
\hline $\boldsymbol{P}_{\boldsymbol{o}}$ & Perimeter of object \\
\hline $\boldsymbol{P}_{\boldsymbol{e}}$ & Equivalent perimeter \\
\hline $\boldsymbol{A}_{\boldsymbol{o}}$ & Area of object \\
\hline $\boldsymbol{A}_{\boldsymbol{e}}$ & Equivalent area \\
\hline $\boldsymbol{E}$ & Ellipseness \\
\hline $\boldsymbol{a}$ & Major radius of ellipse \\
\hline $\boldsymbol{b}$ & Minor radius of ellipse \\
\hline $\boldsymbol{P D F}$ & Probability density function \\
\hline $\boldsymbol{C D F}$ & Cumulative distribution function \\
\hline
\end{tabular}

\section{REFERENCES}

1. Indraratna, B., Ionescu, D. \& Christie, H. 1998. Shear behavior of railway ballast based on large-scale triaxial tests. Journal of Geotechnical and Geoenvironmental Engineering, 124, 439450 .

2. Anderson, W. \& Fair, P. 2008. Behavior of Railroad Ballast under Monotonic and Cyclic Loading. Journal of Geotechnical and Geoenvironmental Engineering, 134, 316.

3. Aursudkij, B., McDowell, G. R. \& Collop, A. C. 2009. Cyclic loading of railway ballast under triaxial conditions and in a railway test facility. Granular Matter, 11, 391 to 401. 
4. Cho, G.-C., Dodds, J. \& Santamarina, J. C. 2006. Particle Shape Effects on Packing Density, Stiffness and Strength: Natural and Crushed Sands. Journal of Geotechnical and Geoenvironmental Engineering, ASCE, 132, 591 to 602.

5. Marachi, N. D., Chan, C. K. \& Seed, H. B. (1972) Evaluation of Properties of Rockfill Materials. Journal of the Soil Mechanics and Foundations Division, Proceedings of the American Society of Civil Engineers, Vol.98, pp.95-114.

6. Sevi, A. F. 2008. Physical Modeling Of Railroad Ballast Using The Parallel Gradation Scaling Technique Within The Cyclical Triaxial Framework. Doctor of Philosophy PhD thesis, Missouri University of Science and Technology.

7. Harkness, J. 2009. Potential particles for the modelling of interlocking media in three dimensions. International Journal For Numerical Methods In Engineering, Volume 80, 1573 to 1594.

8. Zeller, J. \& Wullimann, R., 1957. The shear strength of the shell materials for the GoSchenenalp Dam, Switzerland. In Proc., 4th Inst. J on SMFE. London, p. 399-404.

9. Lowe, John, 1964. Shear Strength of Coarse Embankment Dam Materials. Proceedings, 8th Congress on Large dams, pp. 745-761.

10. Varadarajan, A., Sharma, K. G., Venkatachalam, K. \& Gupta, A. K. 2003. Testing and Modeling Two Rockfill Materials. Journal of Geotechnical and Geoenvironmental Engineering, ASCE, 129, 203 to 218.

11. McDowell, G. R. 2001. Statistics of soil particle strength. Geotechnique, 51, 90.

12. Frossard, E., Hu, E., Dano, C. \& Hicher, P.-Y. 2012. Rockfill shear strength evaluation: a rational method based on size effects. Geotechnique, 62, 415 to 427.

13. Hertz, H. R. 1896. Miscellaneous Papers, London, MacMillan and Co. Ltd, New York, Macmillan and Co.

14. Johnson, K. L. 1985. Contact mechanics, Cambridge, Cambridge University Press.

15. Cavarretta, I., Coop, M. \& O'sullivan, C. 2010. The influence of particle characteristics on the behaviour of coarse grained soils. Geotechnique, 60, 413 to 423.

16. Abbireddy, C. 2008. Particle Form and its Impact on Packing and Shear Behaviour of Particulate Materials. Doctor of Philosophy, University of Southampton.

17. Barrett , P. J. 1980. The shape of rock particles, a critical review. Sedimentology, 27, 291-303. 18. Wadell, H. (1932) Volume, shape and roundness of rock particles. J. Geol., Vol.40, pp.443 to 451.

19. Francus, P. (ed.) 2004. Image Analysis, Sediments and Paleoenvironments, Volume 7, The Netherlands: Springer.

20. Erdogan, S. T., Quiroga, P. N., Fowler, D. W., Saleh, H. A., Livingston, R. A., Garboczi, E. J., Ketcham, P. M., Hagedorn, J. G. \& Satterfield, S. G. 2006. Three-dimensional shape analysis of coarse aggregates: New techniques for and preliminary results on several different coarse aggregates and reference rocks. Cement and Concrete Research, Vol. 36, pp 1619 to 1627. 21. Masad, E., Saadeh, S., Al-Rousan, T., Garboczi, E. \& Little, D. 2005. Computations of particle surface characteristics using optical and X-ray CT images. Computational Materials Science, 34, 406-424. 
22. Taylor, M. A., Garboczi, E. J., Erdogan, S. T. \& Fowler, D. W. 2006. Some properties of irregular 3-D particles. Powder Technology, Vol. 162, pp. 1 to15.

23. Quiroga, P. N. \& Fowler, D. W. The Effects of Aggregates Characteristics on the Performance of Portland Cement Concrete, Research Report ICAR - 104-1F. International Center for Aggregate Research, ICAR.

24. Zingg, T., 1935. Contribution to the gravel analysis (Beitrag zur Schotteranalyse ).

Schweizminer. Petrog. Mitt, 15(38-140).

25. Blott, S. J. \& Pye, K. (2008) Particle shape: a review and new methods of characterization and classification. Sedimentology, Vol.55, pp.31-63.

26. Sneed, E. D. \& Folk, R. L. (1958) Pebbles in the Lower Colorado River, Texas, a study in particle morphogenesis. J. Geol., Vol.66, pp.114 to 150.

27. Pirard, E. (2005). Image Measurements. In: FRANCUS, P. (ed.) Image Analysis, Sediments and Paleoenvironments. Kluwer Academic Publishers, Dordrecht, The Netherlands.

28. Clayton, C. R. I., Abbireddy, C. O. R., \& Schiebel, R. 2009. A method of estimating the form of coarse particulates. Geotechnique, 59, Issue 6, 493 to 501.

29. Garboczi, E. J. 2002. Three-dimensional mathematical analysis of particle shape using X-ray tomography and spherical harmonics: Application to aggregates used in concrete. Cement and Concrete Research, Vol. 32(10), pp. 1621 to 1638.

30. Krumbein, W. C. (1941) Measurement and geological significance of shape and roundness of sedimentary particles. J. sedim. Petrol, Vol.11, pp.64 to 72.

31. Krumbein, W. C. \& Sloss, L. L. 1963. Stratigraphy and Sedimentation, W. H. Freeman and company, San Francisco and London.

32. Pirard, E. 1994. Shape processing and analysis using the calypter. Journal of Microscopy, Vol. 175, Pt 3, pp. 214 to 221.

33. Mediacy (2011) Image Pro Plus webpage: http://www.mediacy.com/index.aspx?page=IPP accessed March 2011.

34. Ramanujan, S. 1913-1914. Modular Equations and Approximations to $\pi$. Quarterly Journal of Pure and Applied Mathematics, Vol. 45 pp. 350 to 372.

35. Almkvist, G. \& Berndt, B. 1988. Gauss, Landen, Ramanujan, the arithmeticgeometric mean, ellipses, and the Ladies Diary. The American Mathematical Monthly, Vol. 95, pp. 585 to 608. 36. Corder, Gregory W., and Dale I. Foreman.(2009) Nonparametric Statistics for NonStatisticians. 1st ed. Hoboken: John Wiley \& Sons, Inc pages 99-105

37. Weibull, W. 1951. A Statistical Distribution Function of Wide Applicability. Journal of Applied Mechanics, 18, 293-297.

38. Mathworks. 2012. Matlab [Online]. Available:http://www.mathworks.co.uk/products/matlab/ [Accessed May 2012].

39. Houlsby, G. T. 2009. Potential particles: a method for modelling non-circular particles in DEM. Computers and Geotechnics, Volume 36, Issue 6, Pages 953-959.

40. Radjaï, F. \& Dubois, F. (eds.) March 2011. Discrete Numerical Modeling of granular materials: Hardcover, Wiley-Iste, ISBN 978-1-84821-260-2. 


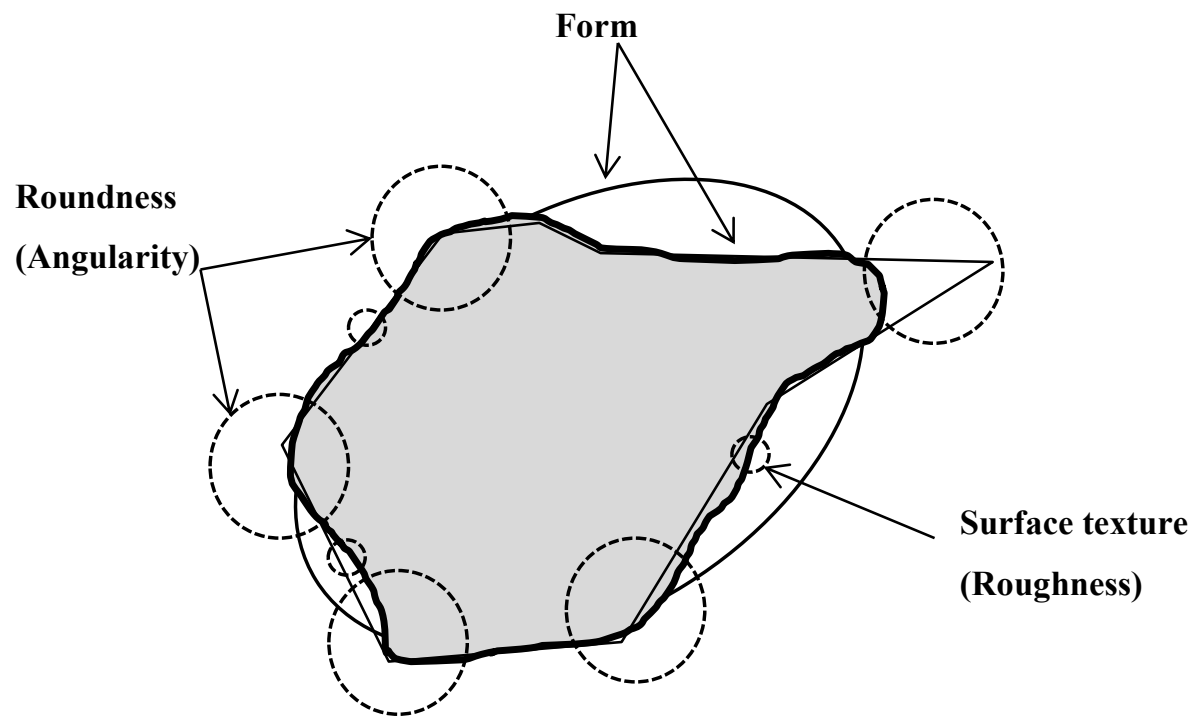

Figure 1: Properties of particle shape (after Barrett, 1980)

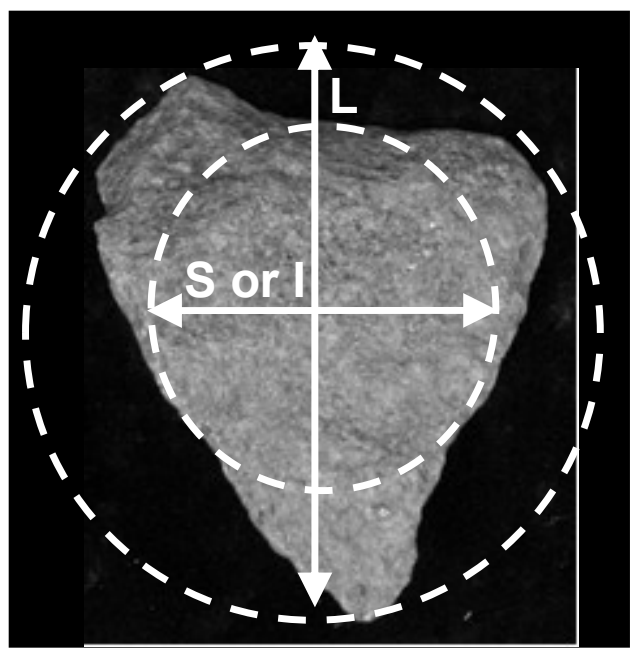

Figure 2: Form indicators 


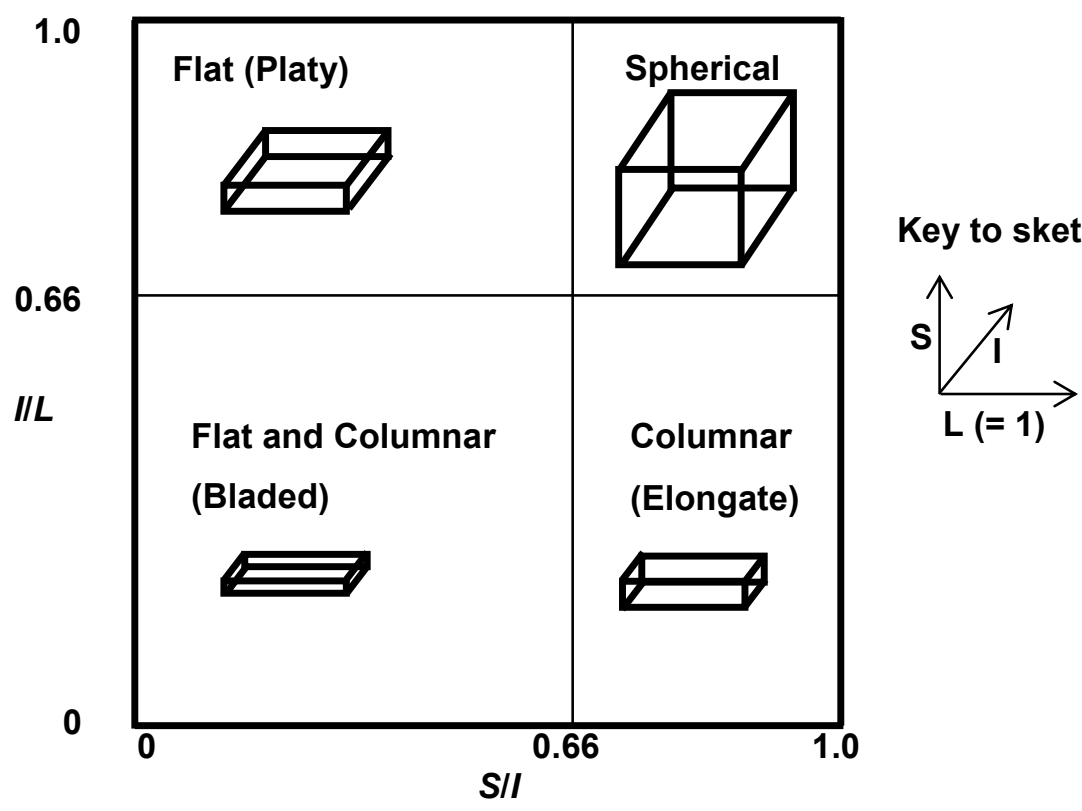

Figure 3: Zingg plot to characterize particle form (redrawn from Zingg, 1935, alternative descriptors in brackets selected from Blott and Pye, 2008)

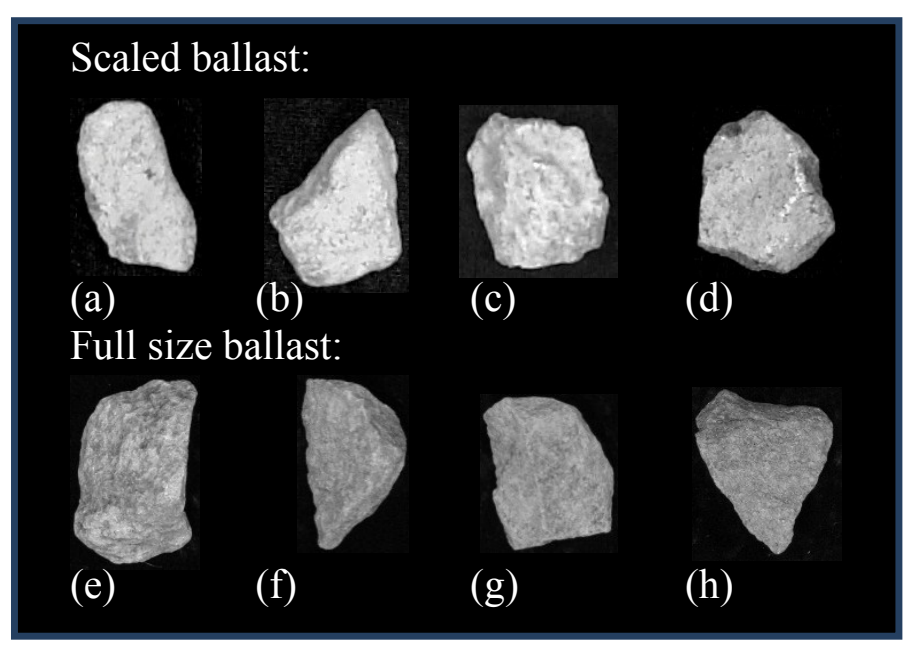

Figure 4: Example particles in sieve intervals (mm): (a) 9.5 to 11.2 (b) 11.2 to 13.2 (c) 13.2 to 16.0, (d) 16.0 to 22.4 (e) 22.4 to 31.5 (f) 31.5 to 40.0 (g) 40.0 to 50.0 (h) 50.0 to 62.5

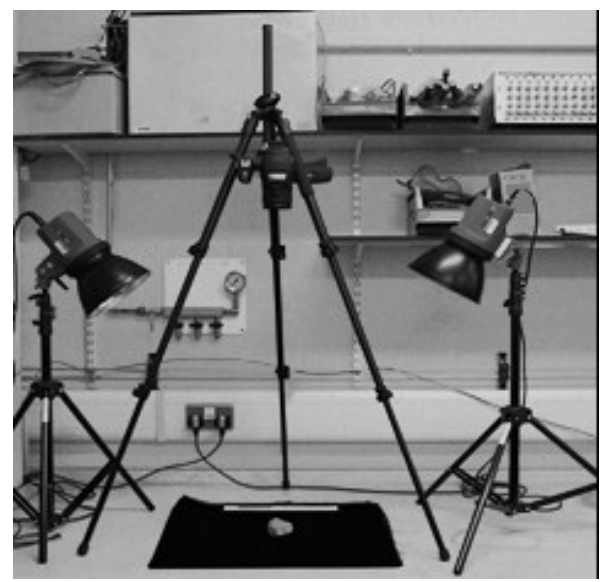

Figure 5: Image capture setup 


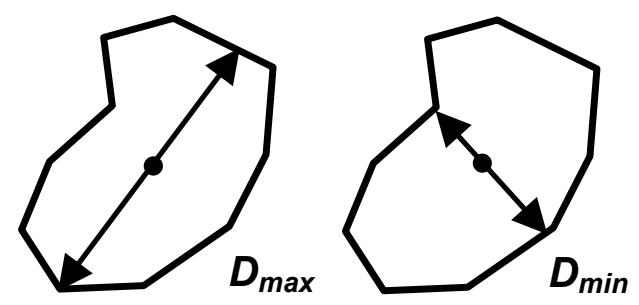

- = Centroid of area

Figure 6: Definitions of $\boldsymbol{D}_{\max }$ and $\boldsymbol{D}_{\min }$
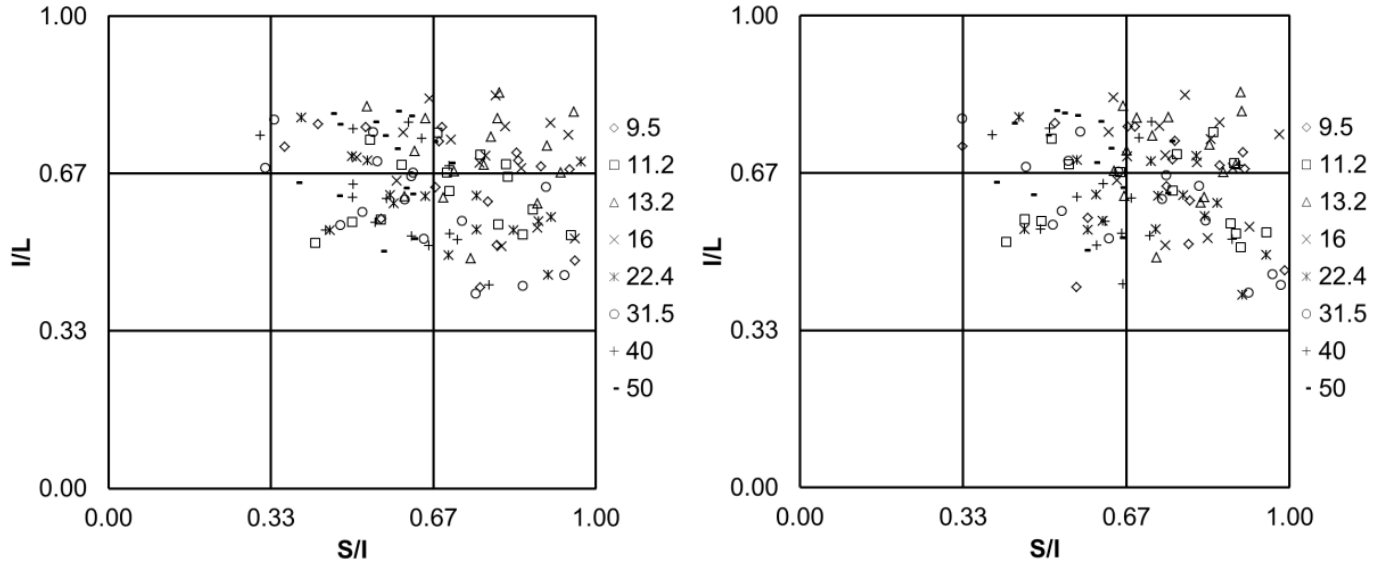

Figure 7: The legend shows the catching sieve in mm (a) Zingg plot for SEES data (b) Zingg plot for data obtained using two orthogonal views

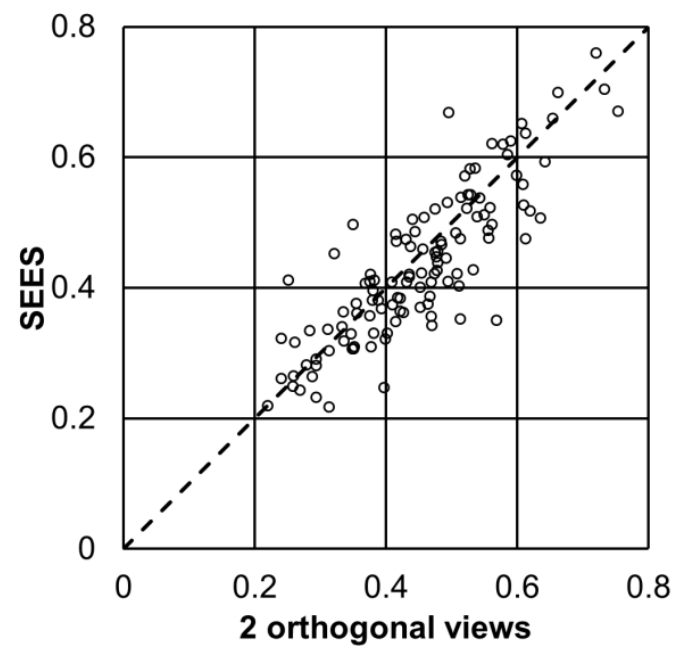

Figure 8: Comparison of $\boldsymbol{S} / \boldsymbol{L}$ ratio obtained from the two different methods (total number of particles evaluated $=120$ ) 

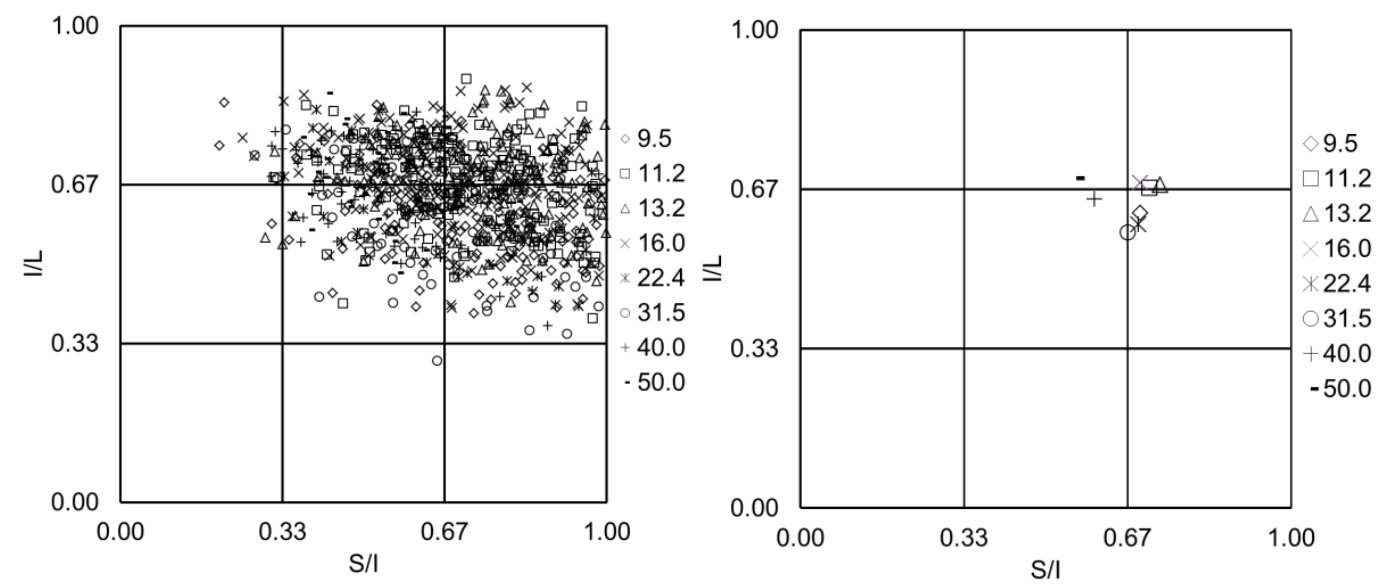

Figure 9 The legend shows the catching sieve in mm, Zingg plots all SEES data for: (a) individual particles (b) averages

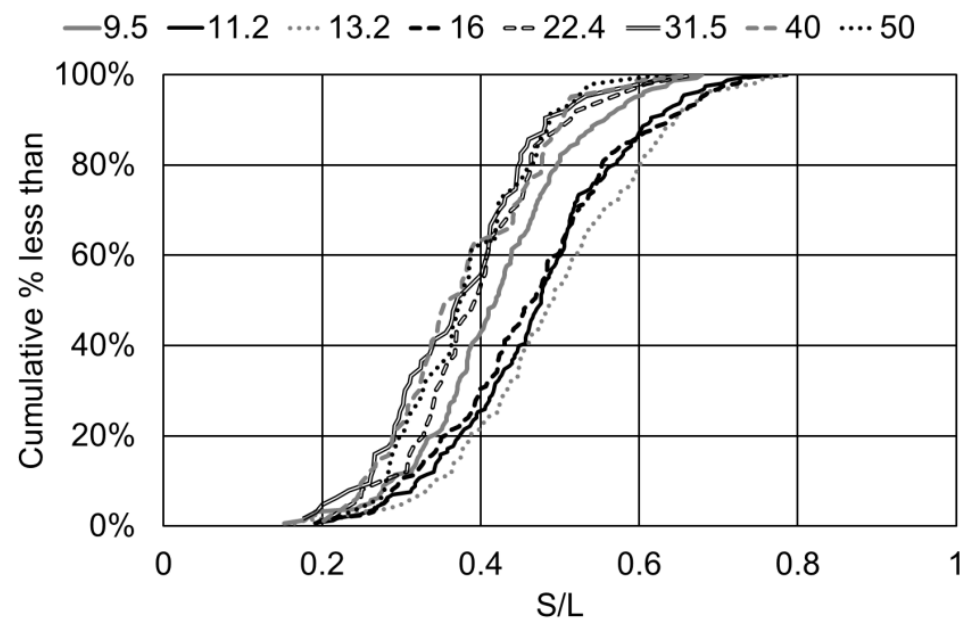

Figure 10: Cumulative distribution curves for $S / L$, the legend shows the catching sieve in $\mathrm{mm}$

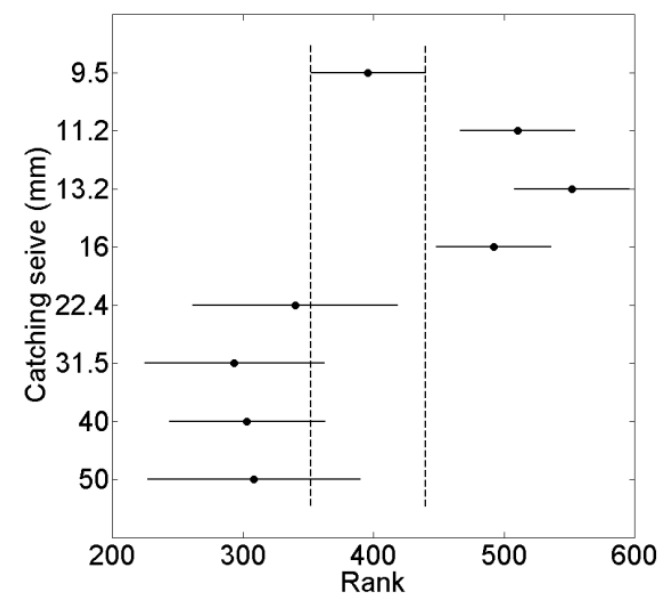

Figure 11: Kruskal Wallis evaluation of form 


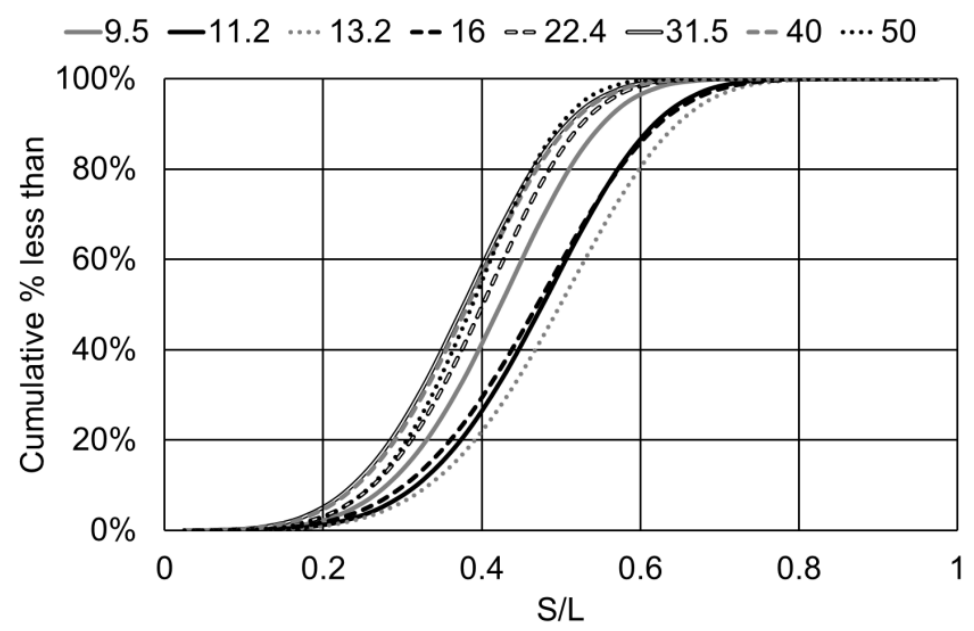

Figure 12: Weibull $\mathrm{CDF}$ for $\mathrm{S} / \mathrm{L}$, the legend shows the catching sieve in $\mathrm{mm}$

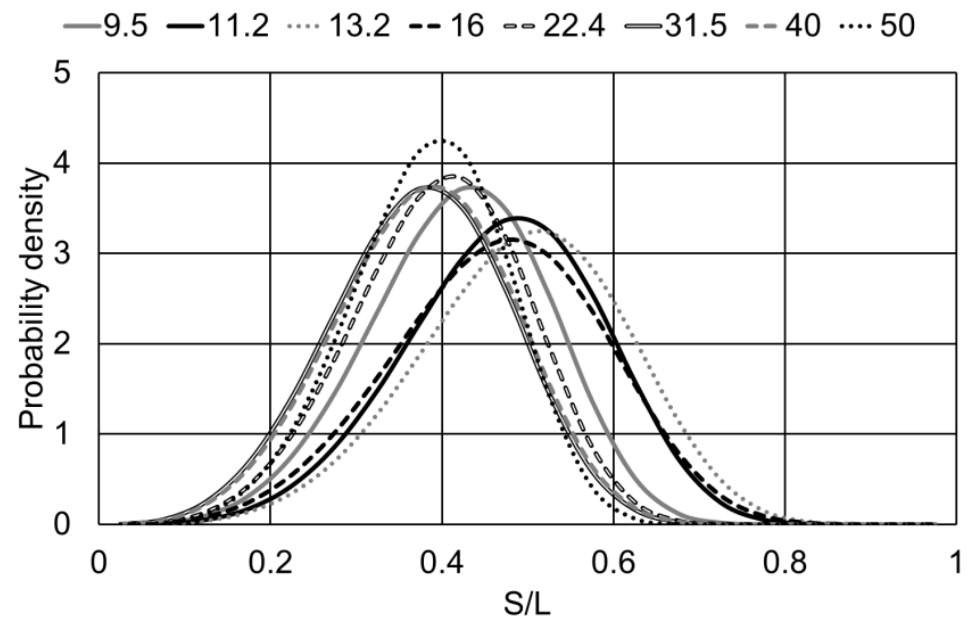

Figure 13: Weibull PDF for S/L, the legend shows the catching sieve in $\mathrm{mm}$

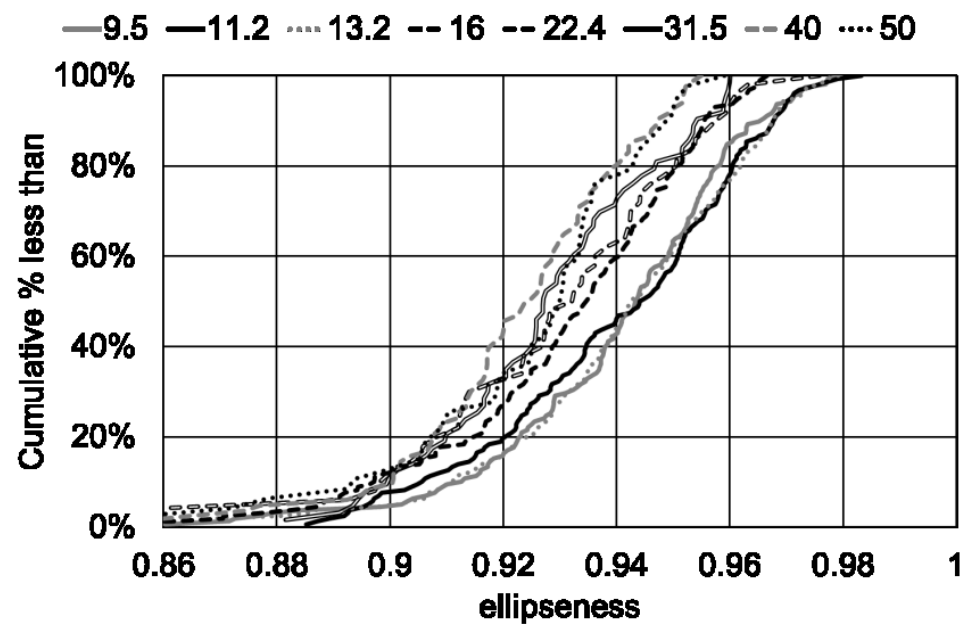

Figure 14: Cumulative distributions, Ellipseness, the legend shows the catching sieve in $\mathrm{mm}$ 


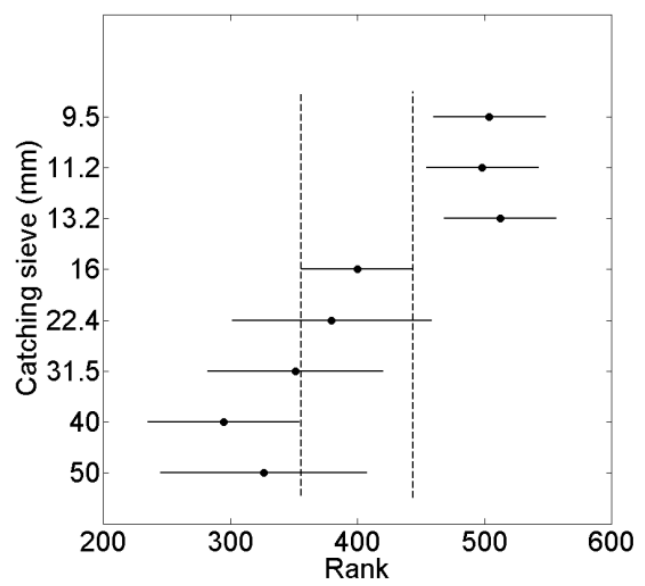

Figure 15: Kruskal Wallis evaluation of ellipseness

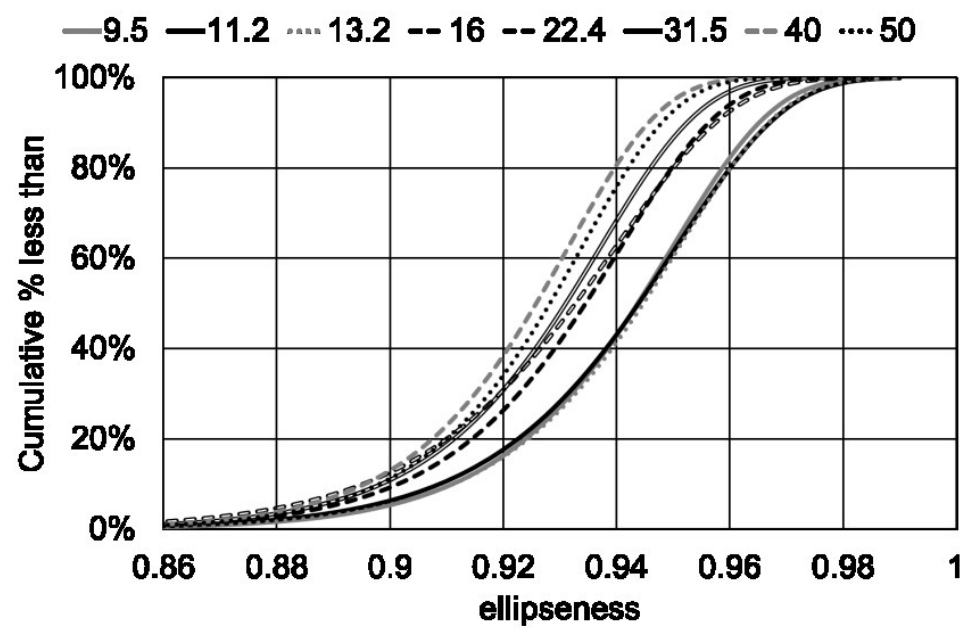

Figure 16: Weibull cumulative distribution functions for ellipseness, the legend shows the catching sieve in $\mathrm{mm}$ 


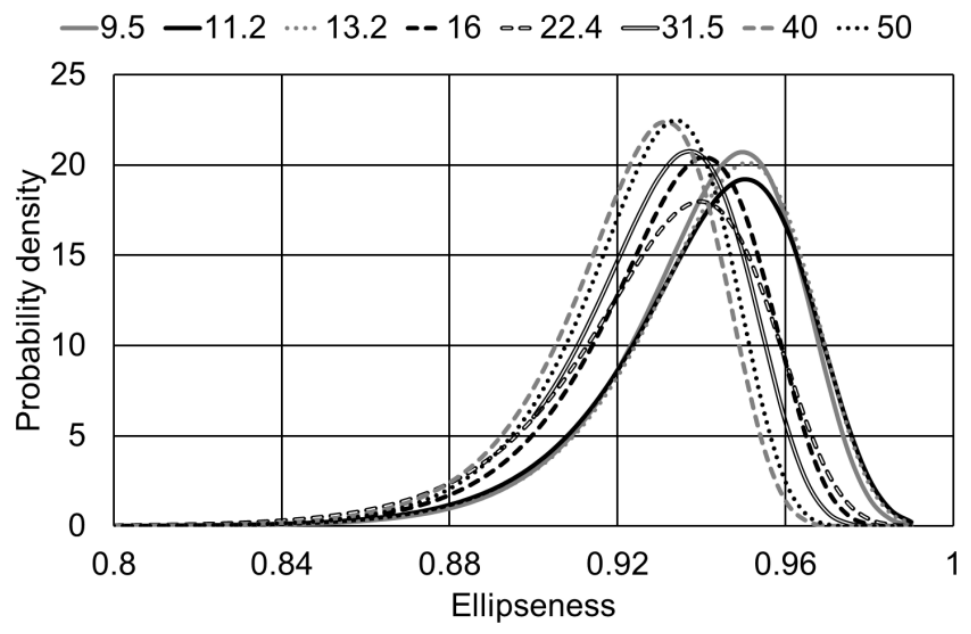

Figure 17: Weibull PDF for ellipseness, the legend shows the catching sieve in $\mathrm{mm}$

Table 1: Basic particle data and comparison between $\mathrm{S} / \mathrm{L}$ values obtained using two orthogonal views and SEES

\begin{tabular}{|l|l|l|l|l|l|l|l|l|}
\hline & \multicolumn{9}{|l|}{ Catching Sieve (mm) } \\
\cline { 2 - 9 } & 9.5 & 11.2 & 13.2 & 16 & 22.4 & 31.5 & 40 & 50 \\
\hline Number of particles & 15 & 15 & 15 & 15 & 15 & 15 & 15 & 15 \\
\hline $\begin{array}{l}\text { Average particle } \\
\text { mass }(\boldsymbol{W})\end{array}$ & 2.43 & 3.76 & 4.56 & 8.67 & 42.04 & 99.46 & 165.95 & 199.17 \\
\hline Average SEES $\boldsymbol{S} / \boldsymbol{L}$ & 0.46 & 0.44 & 0.53 & 0.53 & 0.41 & 0.36 & 0.36 & 0.39 \\
\hline $\begin{array}{l}\text { Average 2 View } \\
\boldsymbol{S} / \boldsymbol{L}\end{array}$ & 0.47 & 0.45 & 0.54 & 0.54 & 0.42 & 0.39 & 0.40 & 0.40 \\
\hline$\%$ difference $\boldsymbol{S} / \boldsymbol{L}$ & $-2.2 \%$ & $-1.6 \%$ & $-1.7 \%$ & $-1.7 \%$ & $-1.5 \%$ & $-7.7 \%$ & $-10.2 \%$ & $-2.0 \%$ \\
\hline
\end{tabular}

Table 2: Particles for image analysis using the plan view of the most stable face (SEES)

\begin{tabular}{|l|l|l|l|l|l|l|l|l|}
\hline & \multicolumn{9}{|l|}{ Catching Sieve (mm) } \\
\cline { 2 - 10 } & 9.5 & 11.2 & 13.2 & 16 & 22.4 & 31.5 & 40 & 50 \\
\hline No. of particles & 158 & 158 & 158 & 158 & 50 & 63 & 83 & 47 \\
\hline Average mass $(\mathrm{g})$ & 2.31 & 3.31 & 3.38 & 8.78 & 42.02 & 100.96 & 136.64 & 192.79 \\
\hline Average $\boldsymbol{S}(\mathrm{mm})$ & 7.8 & 9.2 & 9.5 & 12.5 & 19.9 & 25.9 & 27.9 & 30.7 \\
\hline Average $\boldsymbol{I}(\mathrm{mm})$ & 11.4 & 13.1 & 13.1 & 18.4 & 29.4 & 39.5 & 47.5 & 55.3 \\
\hline Average $\boldsymbol{L}(\mathrm{mm})$ & 18.9 & 20.0 & 19.6 & 27.5 & 50.5 & 71.0 & 74.9 & 81.7 \\
\hline
\end{tabular}


Table 3: Summary data for $I / L, S / I$ and $S / L$

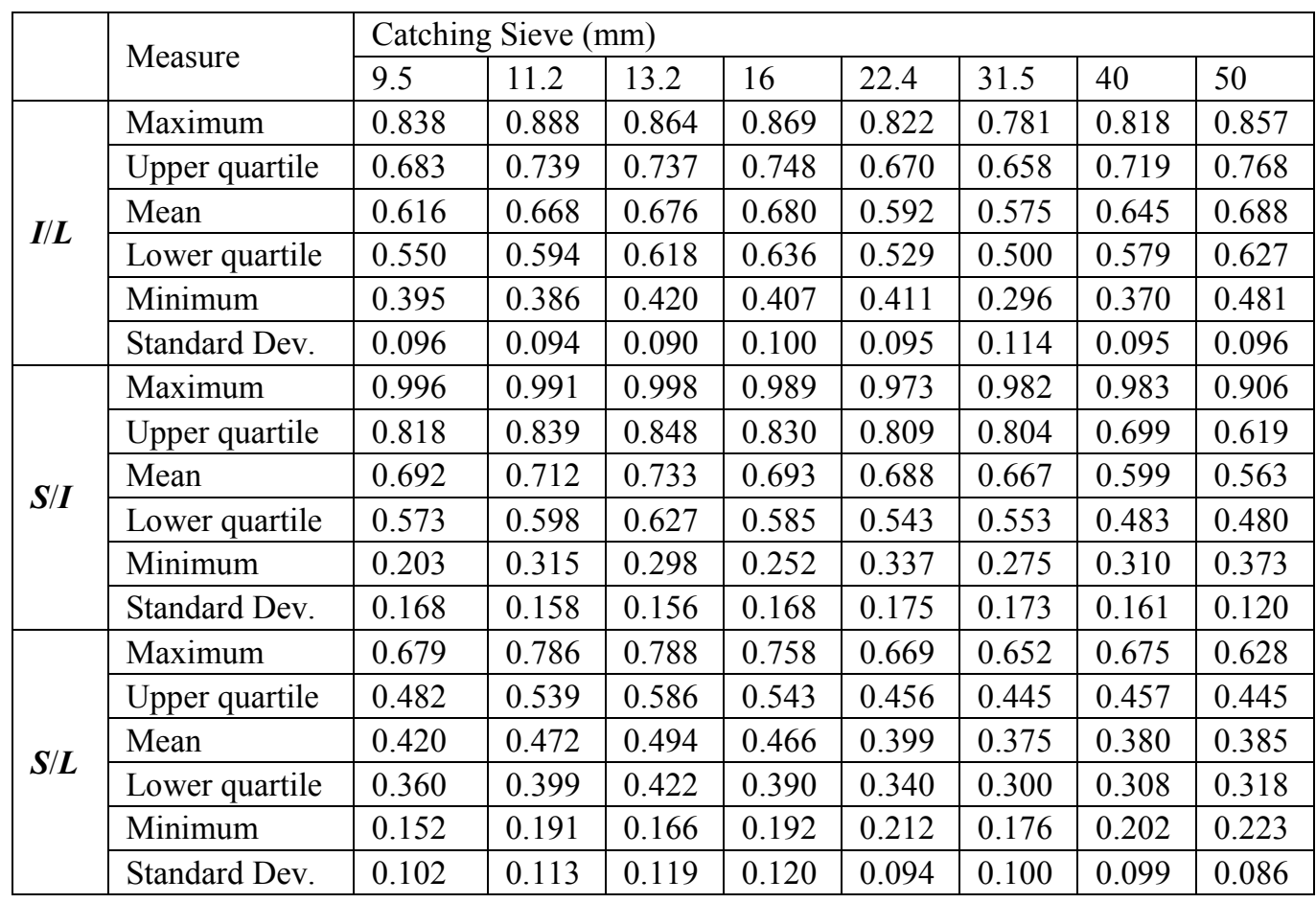

Table 4: Weibull parameters for $\boldsymbol{S} / \boldsymbol{L}$

\begin{tabular}{|c|c|c|c|c|c|c|}
\hline \multirow{3}{*}{$\begin{array}{l}\text { Catching } \\
\text { sieve }(\mathrm{mm})\end{array}$} & \multicolumn{4}{|c|}{$95 \%$ confidence intervals } & \multicolumn{2}{|l|}{ Best fit } \\
\hline & \multicolumn{2}{|l|}{ lower } & \multicolumn{2}{|l|}{ Upper } & & \\
\hline & $a$ & $b$ & $a$ & $b$ & $a$ & $b$ \\
\hline 9.5 & 0.4431 & 4.0420 & 0.4764 & 5.1166 & 0.4594 & 4.5477 \\
\hline 11.2 & 0.4979 & 4.1261 & 0.5345 & 5.2298 & 0.5159 & 4.6453 \\
\hline 13.2 & 0.5213 & 4.1258 & 0.5595 & 5.2450 & 0.5401 & 4.6519 \\
\hline 16 & 0.4930 & 3.7872 & 0.5326 & 4.8047 & 0.5124 & 4.2657 \\
\hline 22.4 & 0.4080 & 3.6368 & 0.4656 & 5.4363 & 0.4358 & 4.4464 \\
\hline 31.5 & 0.3874 & 3.3777 & 0.4406 & 4.8778 & 0.4131 & 4.0591 \\
\hline 40 & 0.3956 & 3.5155 & 0.4418 & 4.8426 & 0.4180 & 4.1260 \\
\hline 50 & 0.3932 & 3.8556 & 0.4467 & 5.8656 & 0.4191 & 4.7555 \\
\hline
\end{tabular}

Table 5: Summary statistics for ellipseness data

\begin{tabular}{|l|l|l|l|l|l|l|l|l|}
\hline \multirow{2}{*}{ Measure } & \multicolumn{9}{|l|}{ Catching Sieve $(\mathrm{mm})$} \\
\cline { 2 - 9 } & 9.5 & 11.2 & 13.2 & 16 & 22.4 & 31.5 & 40 & 50 \\
\hline Maximum & 0.980 & 0.983 & 0.979 & 0.967 & 0.976 & 0.960 & 0.955 & 0.959 \\
\hline Upper quartile & 0.955 & 0.959 & 0.958 & 0.948 & 0.755 & 0.945 & 0.754 & 0.942 \\
\hline Mean & 0.940 & 0.940 & 0.941 & 0.931 & 0.929 & 0.928 & 0.923 & 0.925 \\
\hline Lower quartile & 0.928 & 0.924 & 0.929 & 0.919 & 0.913 & 0.916 & 0.913 & 0.914 \\
\hline Minimum & 0.860 & 0.885 & 0.858 & 0.836 & 0.855 & 0.882 & 0.854 & 0.854 \\
\hline Standard Dev. & 0.022 & 0.023 & 0.023 & 0.023 & 0.025 & 0.020 & 0.020 & 0.022 \\
\hline
\end{tabular}


Table 6: Weibull parameters for ellipseness

\begin{tabular}{|c|c|c|c|c|c|c|}
\hline \multirow{3}{*}{$\begin{array}{l}\text { Catching } \\
\text { sieve } \\
(\mathrm{mm})\end{array}$} & \multicolumn{4}{|c|}{$95 \%$ confidence limits } & \multicolumn{2}{|l|}{ Best fit } \\
\hline & \multicolumn{2}{|l|}{ Lower } & \multicolumn{2}{|l|}{ Upper } & \multirow[b]{2}{*}{$a$} & \multirow[b]{2}{*}{$\boldsymbol{b}$} \\
\hline & $a$ & $b$ & $a$ & $b$ & & \\
\hline 9.5 & 0.9473 & 47.3840 & 0.9531 & 60.4145 & 0.9502 & 53.504 \\
\hline 11.2 & 0.9477 & 43.9051 & 0.9540 & 56.1763 & 0.9509 & 49.6631 \\
\hline 13.2 & 0.9484 & 46.0117 & 0.9544 & 58.8745 & 0.9514 & 52.0473 \\
\hline 16 & 0.9382 & 46.1520 & 0.9441 & 59.2014 & 0.9411 & 52.2711 \\
\hline 22.4 & 0.9342 & 37.0975 & 0.9462 & 56.9157 & 0.9402 & 45.9503 \\
\hline 31.5 & 0.9329 & 43.7752 & 0.9422 & 64.0007 & 0.9375 & 52.9305 \\
\hline 40 & 0.9281 & 48.0098 & 0.9355 & 67.1111 & 0.9318 & 56.7626 \\
\hline 50 & 0.9293 & 45.4772 & 0.9391 & 71.6689 & 0.9342 & 57.0903 \\
\hline
\end{tabular}

